\title{
Parental perception and guideline awareness of children's lifestyle behaviours at ages 5 to 14 in Singapore
}

Phaik Ling Quah ${ }^{1}{ }_{P h D}$, Benny Kai Guo $\underline{\operatorname{Loo}}{ }^{2}{ }_{M B B S}\left(S^{\prime} p o r e\right)$, Nurul Syaza $\underline{\text { Razali }}{ }^{1}{ }_{B S c}$, Nurul Sakinah $\underline{\text { Razali }}{ }^{1}{ }_{B S c}$, Chin Chye Teo ${ }^{1}{ }$, , Kok Hian $\underline{\text { Tan }}^{1,3}{ }^{1,}$ MBBS (S'pore)

\begin{abstract}
Introduction: There are limited data on the descriptive lifestyle behaviour of school-age children in Singapore.

Methods: A total of 100 parents of children ages 5 to 14 participated in a parents' proxy-reported survey. Frequency of moderate physical activity (PA) and vigorous PA was assessed, while t-tests or chi-square test was used to examine differences between weekdays and weekends for sleep, screen viewing time (SVT) and sedentary behaviour (SB).

Results: Of the 100 children (68\% of Chinese ethnicity, 59\% boys, mean age $9.1 \pm 2.9$ years), $31 \%$ were overweight or obese, with body mass index z-score of $>1$. For moderate and vigorous PA participation in a typical week, $32.0 \%$ and $43.0 \%$, respectively, did not participate, while median (interquartile range) days of participation were 3 (2-3) days/week and 2 (1-3) days/week for a duration of 60 (interquartile range 30-120) minutes/session. When comparing weekends with weekdays, the means (standard deviation) of both SVT and sleep duration were higher on weekends (SVT: 4.1 [2.9] versus 3.3 [3.1] hours/day, $P=0.07$; sleep: 8.8 [1.5] vs 8.3 [1.3] hours/day, $P=0.02$ ), while there were no significant differences for SB. A higher proportion of children had SB of $\geq 10$ hours/day and slept $<8$ hours/day on weekdays compared with on weekends (SB: $23.5 \%$ vs $20.6 \%, P>0.05$; sleep: $18.8 \%$ vs $2.1 \%, P<0.05$ ), while the proportion exceeding SVT of 2 hours/day were higher on weekends than on weekdays $(63.8 \%$ vs $45.4 \%, P=0.03)$. Overall, there was higher parental awareness of sleep guidelines (80.0\%), but lower awareness of PA (51.0\%) and SVT (59.0\%) guidelines.
\end{abstract}

Conclusion: Lifestyle behaviours were suboptimal in Singapore children compared with existing overseas guidelines, indicating a need for an integrated guideline with greater dissemination.

\section{Ann Acad Med Singap 2021;50:695-702}

Keywords: Childhood and adolescents, integrated guideline, lifestyle behaviours, physical activity, screen viewing time, sleep

\section{INTRODUCTION}

Evidence from longitudinal studies in Singapore has suggested that long-term health implications on children stems from less-than-optimal lifestyle behaviours, ${ }^{1-3}$ encompassing physical activity (PA), ${ }^{4}$ sleep ${ }^{5}$ and sedentary behaviour (SB) which includes screen viewing time (SVT) ${ }^{6}$

In recent years, specific combinations of integrated movement behaviours (i.e. sufficient PA, low SB, limited SVT and adequate sleep) known as the 24-hour movement guidelines for children 5-17 years were developed in $\mathrm{Canada}^{7}$ and Australia. ${ }^{8}$ The greater the number of integrated guidelines accomplished, the better the health-related quality of life. ${ }^{9}$ These behaviours have also been independently associated with desirable health indicators related to cardiometabolic health ${ }^{4,5,10}$ and better psychosocial health. ${ }^{5,11}$ The recommendations from these guidelines include sufficient PA (e.g. moderate to vigorous $\mathrm{PA}$ of $\geq 60 \mathrm{~min} /$ day), limited SVT ( $\leq 2$ hours/day) and adequate sleep duration (e.g. 9-11 hours/night for children aged 5-13 years). ${ }^{7,8}$ Data of children aged 5.5 years from the Growing Up in Singapore Towards Healthy Outcomes birth cohort study has shown that relatively few children $(5.5 \%)^{12}$ adhered to these guidelines. ${ }^{7,13}$ The study further

\footnotetext{
${ }^{1}$ Division of Obstetrics and Gynaecology, KK Women's and Children's Hospital, Singapore

${ }^{2}$ General Paediatric Service, KK Women's and Children's Hospital, Singapore

${ }^{3}$ Duke-NUS Medical School, Singapore

Correspondence: Prof Kok Hian Tan, Maternal Fetal Medicine, KK Women's and Children's Hospital, 100 Bukit Timah Road, Singapore 229899.

Email: tan.kok.hian@singhealth.com.sg
} 


\section{CLINICAL IMPACT}

\section{What is New}

- This study highlights the suboptimal lifestyle behaviours of the Singaporean children aged 5-14 years.

- Findings also point to the lack of awareness of existing activity guidelines among parents with children in this age group.

\section{Clinical Implications}

- This study led to the development of the Singapore Integrated 24-Hour Activity Guidelines for Children And Adolescents to help with better monitoring and improvement of these behaviours.

suggested the adaption of the integrated movement guidelines for Singapore to better monitor children's daily activities to achieve the desirable health indicators. However, more data on lifestyle behaviours are first required in children from older age groups to support the development of such guidelines.

Currently, most of the data we have on lifestyle behaviours of children in Singapore come from those of preschooler age (3-6 years) $)^{1,3,12,14-16}$ or younger (below 2 years). ${ }^{2,17}$ Cross-sectional data in the preschooler age group have shown that time spent engaged in moderate to vigorous PA is low, while time spent in SB is high: ${ }^{15}$ the duration of night-time sleep is significantly lower than recommended values. ${ }^{16}$ At ages $2-3$ years, $50 \%$ of children were already spending $>2$ hours/day on SVT, exceeding the recommended guidelines for this age group. ${ }^{14}$ Studies in children of ages 5 years and above from Singapore spanning across the school-going age groups (kindergarten, primary and secondary school) are not as comprehensive, especially on evidence surrounding SVT, SB and sleep. Existing studies have focused on either the younger age group only (primary school at ages 7-12) - with only one study on $\mathrm{PA}^{18}$ - and the adolescent age group only (secondary school at ages $13-18$ years), examining $\mathrm{PA}^{19}$ and sleep. ${ }^{20}$

In view of the limited data in children aged 5 years and older, our study aimed to comprehensively assess the lifestyle behaviours of Singapore children aged 5-14 years. Specifically, this survey aimed to (1) describe PA, SVT, SB and sleep; (2) compare SVT, SB and sleep between weekdays and weekends; and (3) describe the parental perceptions of child health and awareness of existing guidelines.

\section{METHODS}

\section{Study sample}

A convenience sampling method was used to recruit participants from the KK Women's and Children's Hospital for this survey. Eligible participants had to be Singaporean or permanent resident parents (mother or father) of children aged 5-14 years, who were able to read and understand English. Parents would need to give verbal consent for an anonymous survey before they were given either a hard copy of the survey to complete or an electronic version via FormSG (https://form.gov.sg/). Parents who completed the survey received SGD25 in cash, and 100 parents completed this survey within a 1-month period from 1 October to 1 November 2020. The research procedures in this study received formal approval for an exempt review by the SingHealth Centralised Institutional Review Board.

\section{Data collection}

The survey comprised 33 items that collected data on demographics, PA, SB, SVT, sleep habits (night-time sleep and day-time napping), parental perceptions on child's health and well-being, and parental awareness of lifestyle guidelines for children and adolescents. The parents would self-report their demographic characteristics and report their child's demographics and lifestyle behaviours by proxy. They were asked to recall their child's past 7-day lifestyle and activities while completing the survey questions. Items on PA and SB were adapted from the International Physical Activity Questionnaire, ${ }^{21}$ while the items on sleep were adapted from the Child Sleep Habits Questionnaire;22 both questionnaires have been widely used to assess PA or sleep. ${ }^{23}$ Recreational SVT was captured as the average time a child spends on a screen. ${ }^{14}$ The entire survey took parents approximately 10-15 minutes to complete.

\section{Assessment of physical activity and sedentary behaviour}

Examples of activities common in children were stated as a guide for parents alongside questions on PA levels: vigorous (i.e. fast running, fast swimming, or fast cycling), moderate (i.e. fast walking or regular-paced cycling) and light (i.e. recreational or leisure walking). Parents reported by proxy their child's SB separately for weekdays and weekends, with SB being defined as time spent at school and at home, including time spent sitting at a desk studying and reading, as well as reclining, sitting or lying down to watch television. ${ }^{24}$ 


\section{Assessment of screen time}

Screen viewing time duration (in hours and minutes) was assessed using the questions "In a typical week, how much time does your child spend on recreational screen viewing time on a weekday?" and "In a typical week, how much time does your child spend on recreational screen viewing time on a weekend?" Screen time included the use of 3 types of screen devices: television (viewing or playing television games), computer and handheld devices.

\section{Assessment of sleep}

Parents reported their child's bedtime, waketime, naptime duration (in hours and minutes) and naptime frequency separately for weekdays and weekends. Bedtimes and waketimes were used to calculate total 24-hour sleep times for each child. Naptime frequency was captured with the question "In the past week, how many naps does your child take per day?", with the response options of "No naps", "1 nap", "2 naps" and "3 naps or more". Naptime duration was captured with the questions "What is the average time spent on a nap during a weekday?" and "What is the average time spent on a nap during a weekend?".

\section{Parental perception of child' health}

Parental perception of their child's weight, calorie intake, PA, screen time exposure and sleep were assessed with the question "Do you think your child is ...", with the response options of "Overweight", "Normal weight" and "Underweight". The other 4 questions were (1) "Do you feel that your child receives adequate amount of physical activity to benefit his/ her growth, development and health?"; (2) "Are you concerned about the amount of recreational screen time your child is currently exposed to?"; (3) "Do you think your child is getting adequate sleep to support his/her growth, development and health?", with the response options of "Yes" and "No"; and (4) "Do you think your child is receiving adequate calories to support his/her growth, development and health?", with the response options of "Yes", "Too much" and "No".

\section{Parental awareness of lifestyle guidelines for children}

To assess parental awareness of available lifestyle guidelines for children, parents were asked 3 separate questions regarding PA, sleep and screen time: "Are you aware of the current recommendations regarding the amount of physical activity/sleep/screen time your child should be receiving per day?", with the response options of "Yes" and "No".

\section{Statistical analyses}

Continuous datasets that were normally distributed were presented as mean and standard deviation (SD), while non-normally distributed datasets were presented as median and interquartile range (IQR). SVT, SB and sleep were analysed as continuous variables and categorical variables. SVT was categorised into 3 levels ( $\leq 2,>2-4$ and $>4$ hours), as evidence has shown that recreational SVT of more than 2 hours daily is associated with the most adverse health outcomes. ${ }^{7,11,25}$ SB was categorised into $<10$ and $\geq 10$ hours, based on reported evidence that $\mathrm{SB}$ taking up more than $60-70 \%$ of one's waking day is considered high SB. ${ }^{26}$ In this survey, 10 hours of SB out of 15 waking hours (considering mean duration of 9 hours of sleep) is about $67 \%$ of a child's day. Sleep was categorised into 3 levels ( $<8,8-9$ and $>9$ hours) based on the recommended amount of sleep for school-aged children of 7-18 years (i.e. $8-9$ hours/day). ${ }^{27}$ The independent t-test and the chi-square test were used to compare continuous and categorical variables, respectively, between the weekday and weekend. Sex- and age-specific body mass index (BMI) z-scores were derived using the World Health Organization (WHO) references. ${ }^{28}$ The cut-offs for overweight and obese were defined as $+1 \mathrm{SD}$ and $+2 \mathrm{SD}$, respectively, above the reference distribution as per WHO recommendations. ${ }^{29}$ Statistically significant results were determined at 2 -sided $P<0.05$. All analyses were performed using the STATA software version 13 (StataCorp, College Station, US).

\section{RESULTS}

\section{Study participants}

Parent and child characteristics are detailed in Table 1. The mean age of the 100 respondents was $40.5 \pm 4.8$ years, with the majority being mothers $(89.0 \%)$. Of all the parents, $68.0 \%$ were Chinese, $62.6 \%$ had a university education and $63.0 \%$ were reporting by proxy for their first child.

Child demographics revealed that $50.9 \%$ were boys, the mean age was $9.1 \pm 2.9$ years, and $92.0 \%$ did not have any chronic illnesses. The mean BMI z-score was $-0.22 \pm 2.27 ; 18.0 \%$ of the children were overweight with a BMI $z$-score of $>1$, and $13 \%$ were obese with a BMI z-score of $>2$.

\section{Parents' proxy-reported physical activity}

Table 2 shows the descriptive characteristics of parents' proxy-reported child engagement in PA. In a typical week, $57.0 \%, 68.0 \%$ and $92.0 \%$ of children participated 
Table 1. Characteristics of the parent respondents of the survey and their children $(\mathrm{N}=100)$

\begin{tabular}{|l|c|}
\hline Characteristics & $\begin{array}{c}\text { No. (\%) or mean } \pm \text { standard } \\
\text { deviation }\end{array}$ \\
\hline Respondent characteristics & \\
\hline Parent & $89(89.0)$ \\
\hline Mother & $11(11.0)$ \\
\hline Father & $40.5 \pm 4.8$ \\
\hline Age, year & \\
\hline Ethnicity & $68(68.0)$ \\
\hline Chinese & $25(25.0)$ \\
\hline Malay & $7(7.0)$ \\
\hline Indian & \\
\hline Education & \\
\hline University & $62(62.6)$ \\
\hline Secondary & $31(31.3)$ \\
\hline Primary & $6(6.1)$ \\
\hline
\end{tabular}

\section{Child characteristics}

\begin{tabular}{|lc|}
\hline Birth order & $63(63.0)$ \\
\hline First-born & $37(37.0)$ \\
\hline Second-born or later-born & \\
\hline Sex & $59(59.0)$ \\
\hline Male & $41(41.0)$ \\
\hline Female & \\
\hline Chronic illness & \\
\hline No & $92(92.0)$ \\
\hline Yes & $8(8.0)$ \\
\hline Age (year) & $9.1 \pm 2.9$ \\
\hline Body mass index $(z \text {-scores })^{\mathrm{c}}$ & $-0.22 \pm 2.27$ \\
\hline Overweight $\left(>+1\right.$ z-score $^{\mathrm{c}}$ & $18(18.0)$ \\
\hline Obese $(>+2 \text { z-score })^{\mathrm{c}}$ & $13(13.0)$ \\
\hline
\end{tabular}

${ }^{\text {a }}$ Missing data, $\mathrm{n}=1$

${ }^{\mathrm{b}}$ Chronic illness includes asthma, diabetes, congenital heart disease, cerebral palsy, sickle cell anaemia, spina bifida and epilepsy

c Sex- and age-specific body mass index z-scores were derived using the World Health Organization references

in vigorous PA, moderate PA and low PA, respectively. Median days of children participating in vigorous PA and moderate PA in a typical week were 3 (IQR 2-3) days/week and 2 (IQR 1-3) days/week for a duration of 60 (IQR 30-120) minutes/session, respectively. The median frequency of low PA
Table 2. Parents' proxy-reported engagement in physical activity, frequency per week and duration per session of vigorous, moderate and light physical activity in children aged 5-14 years

\begin{tabular}{|c|c|}
\hline Physical activity & No. $(\%)$ or median (IQR) \\
\hline \multicolumn{2}{|l|}{ Vigorous } \\
\hline Yes & $57(57.0)$ \\
\hline Frequency per week (day) & $3(2-3)$ \\
\hline Duration per session (min) & $60(30-120)$ \\
\hline No & $43(43.0)$ \\
\hline \multicolumn{2}{|l|}{ Moderate } \\
\hline Yes & $68(68.0)$ \\
\hline Frequency per week (day) & $2(1-3)$ \\
\hline Duration per session (min) & $60(30-120)$ \\
\hline No & $32(32.0)$ \\
\hline \multicolumn{2}{|l|}{ Light } \\
\hline Yes & $92(92.0)$ \\
\hline Frequency per week (day) & $5(3-7)$ \\
\hline Duration per session (min) & $30(20-60)$ \\
\hline No & $8(8.0)$ \\
\hline
\end{tabular}

IQR: interquartile range

engagement was 5 (IQR 3-7) days/week for 30 (IQR 20-60) minutes/session.

\section{Parents' proxy-reported SVT, SB and sleep}

Table 3 shows the SVT, SB and sleep duration assessed in the children, comparing weekdays with weekends. Most children were engaged in SVT on weekdays $(90 \%)$ and weekends (94\%), and the children had, on average, higher SVT on weekends than on weekdays (4.1 \pm 2.9 vs $3.3 \pm 3.1$ hours/day; $P=0.07)$. Similarly, the percentage of children exceeding 2 hours/day of SVT (i.e. $>2-4$ and $>4$ hours/day) tended to be higher on weekends (29.8\% and $34.0 \%$, respectively) than on weekdays (25.6\% and $19.8 \%$, respectively; $P=0.03$ ). In contrast, the overall average time spent in $\mathrm{SB}$ was higher on weekdays than on weekends $(6.5 \pm 3.3$ vs $6.0 \pm 3.1$ hours/day, $P=0.26$ ), with a higher percentage of children spending more time in high SB $(\geq 10$ hours/day) on weekdays than on weekends $(23.5 \%$ vs $20.6 \%, P=0.23)$. Children in this survey had less night-time sleep on weekdays than on weekends ( $8.3 \pm 1.3$ vs $8.8 \pm 1.5$ hours/day, $P=0.02)$, with $18.8 \%$ of children receiving less than 8 hours of sleep on weekdays compared with only $2.1 \%$ of children on weekends $(P=0.001)$. 
Table 3. Parents' proxy-reported screen viewing time, sedentary behaviour and sleep in children aged 5-14 years ${ }^{\mathrm{a}}$

\begin{tabular}{|c|c|c|c|}
\hline & Weekday & Weekend & $P$ value \\
\hline \multicolumn{4}{|l|}{ Screen time, hour/day ${ }^{\mathrm{b}}$} \\
\hline$\leq 2$ & $47(54.6)$ & $34(36.2)$ & \\
\hline$>2-4$ & $22(25.6)$ & $28(29.8)$ & \\
\hline$>4$ & $17(19.8)$ & $32(34.0)$ & \\
\hline Mean \pm SD & $3.3 \pm 3.1$ & $4.1 \pm 2.9$ & 0.07 \\
\hline Sedentary behaviour, hour/day ${ }^{c}$ & & & 0.23 \\
\hline$<10$ & $75(76.5)$ & $77(79.4)$ & \\
\hline$\geq 10$ & $23(23.5)$ & $20(20.6)$ & \\
\hline Mean \pm SD & $6.5 \pm 3.3$ & $6.0 \pm 3.1$ & 0.26 \\
\hline Night-time sleep, hour/day ${ }^{\mathrm{d}}$ & & & 0.001 \\
\hline$<8$ & $18(18.8)$ & $2(2.1)$ & \\
\hline $8-9$ & $45(46.9)$ & $31(31.6)$ & \\
\hline$>9$ & $33(34.4)$ & $65(66.3)$ & \\
\hline Mean \pm SD & $8.3 \pm 1.3$ & $8.8 \pm 1.5$ & 0.02 \\
\hline
\end{tabular}

SD: standard deviation

${ }^{a}$ Data are presented as no. (\%) or mean \pm standard deviation

b Weekday: $n=90$, with 4 missing; weekend: $n=94$

c Missing data: $n=2$ for weekday, $n=3$ for weekend

${ }^{\mathrm{d}}$ Missing data: $\mathrm{n}=4$ for weekday, $\mathrm{n}=2$ for weekend

\section{Parental perception of child health and awareness of existing lifestyle behaviour guidelines}

Table 4 shows parental perceptions of their child's health. Of the parents, $20.0 \%$ and $18.0 \%$ perceived their child to be underweight and receiving inadequate calories, respectively, while $11.0 \%$ perceived their child to be overweight and receiving too much calories. There were $37.0 \%$ and $34.0 \%$ of parents who perceived their child to be receiving inadequate PA and sleep, respectively, while $73.0 \%$ expressed concerns over their child's SVT. Only $51.0 \%$ and $59.0 \%$ of parents were aware of existing PA and SVT guidelines, respectively, whereas $80.0 \%$ were aware of existing sleep guidelines.

\section{DISCUSSION}

This study gave us further insight into the lifestyle behaviours of children aged 5-14 years. Our data revealed that in a typical week, approximately $30 \%$ and $40 \%$ of the children in this age group did not engage in any vigorous or moderate $\mathrm{PA}$, respectively. At least a fifth were engaged in high SB and were not receiving adequate sleep, while more than half were exceeding SVT recommendations. None of the children in this study met the recommended PA guidelines, while only
$19.3 \%$ and $21.7 \%$ met both sleep and SVT guidelines on weekdays and weekends, respectively (data not shown). ${ }^{7}$

Children who engaged in PA spent only 2-3 days/week in moderate or vigorous PA. Our observations of low PA involvement concur with another Singapore study using self-reported PA from primary school students. ${ }^{18}$ However, other Singapore studies have shown that engagement in PA was substantially lower when measured using heart rate monitoring data, with $<15 \%$ of the primary school children ${ }^{18,30}$ and none of the adolescents ${ }^{19}$ meeting recommended PA guidelines. This trend of relatively low PA seems to begin as early as preschool; results from accelerometery data showed that children in Singapore aged 4.4 years spent only a median of 0.5 (interquartile range $0.3-0.8$ ) hour/day on moderate to vigorous PA. ${ }^{15}$ A large 12-country study (including 8 Western, 2 Asian and 2 African countries) using data from accelerometers reported that the overall adherence to moderate to vigorous PA guidelines in other Western and Asian countries in children of primary school age (9-11 years) was $44 \% .{ }^{31}$ The highest adherence was in Finland at $61 \%$, and the lowest adherence was in China at $15 \%$. Our findings, which included other existing Singapore-published data, ${ }^{12,18,19,30}$ 
Table 4. Parental perception on child health and parental awareness of available lifestyle guidelines $(\mathrm{N}=100)$

\begin{tabular}{|c|c|}
\hline Parental perceptions & No. $(\%)$ \\
\hline \multicolumn{2}{|l|}{ Weight of child } \\
\hline Normal & $69(69.0)$ \\
\hline Underweight & $20(20.0)$ \\
\hline Overweight & $11(11.0)$ \\
\hline \multicolumn{2}{|c|}{ Child receiving adequate physical activity } \\
\hline Yes & $63(63.0)$ \\
\hline No & $37(37.0)$ \\
\hline \multicolumn{2}{|c|}{ Concerns over screen viewing time of child } \\
\hline Yes & $73(73.0)$ \\
\hline No & $27(27.0)$ \\
\hline \multicolumn{2}{|c|}{ Child receiving adequate sleep } \\
\hline Yes & $66(66.0)$ \\
\hline No & $34(34.0)$ \\
\hline \multicolumn{2}{|c|}{ Child receiving adequate calories } \\
\hline Yes & $71(71.0)$ \\
\hline No & $18(18.0)$ \\
\hline Yes, too much & $11(11.0)$ \\
\hline \multicolumn{2}{|c|}{ Awareness of physical activity guidelines } \\
\hline Yes & $51(51.0)$ \\
\hline No & $49(49.0)$ \\
\hline \multicolumn{2}{|c|}{ Awareness of sleep guidelines } \\
\hline Yes & $80(80.0)$ \\
\hline No & $20(20.0)$ \\
\hline \multicolumn{2}{|c|}{ Awareness of screen time guidelines } \\
\hline Yes & $59(59.0)$ \\
\hline No & $41(41.0)$ \\
\hline
\end{tabular}

point to overall low PA involvement in all age groups and indicate that any interventions promoting PA in children need to begin as early as preschool. With great emphasis placed on homework, enrichments classes and long hours spent in school, more opportunities for movement and play should be incorporated into the school-based programmes.

Time spent watching television remains the most common measure of SB in children and adolescence. ${ }^{25}$ Our findings of SVT, which ranged from 3-4 hours/ day in children with a mean age of 9 years, agree with a Singapore-published survey, ${ }^{32}$ and weekends seem to be the time when more children indulge in recreational screen time, as previously shown. ${ }^{33,34}$ It is known that higher SVT is common in higher-income countries $^{31}$ like Singapore, where handheld devices have become increasingly accessible to young children and where most adults own their own screen devices..$^{35}$ The average SVT reported in our study was comparable to data in children aged 9-11 years from higher-income countries like the UK, US and Australia, with SVT ranging from 3.0 to 3.4 hours/day. ${ }^{31}$ Excessive SVT appears to begin in early childhood, with $75 \%$ of Singaporean children aged 2 years exceeding the American Academy of Pediatrics recommendations of 1 hour/day limit at age 2 years, ${ }^{14}$ once again alluding that interventions need to begin at preschool level or earlier to reduce children's SVT at a later age.

Interestingly, children in our study were engaged in longer durations of SB, with a higher proportion in high SB ( $>10$ hours/day) during the weekdays than on weekends. These observations suggest that SVT might not be the key contributor to SB in our population. ${ }^{36}$ Especially in Singapore, the schooling culture leans towards prolonged sitting time during lessons, followed by a very constructed after-school life filled with enrichment classes or homework that would altogether contribute to longer durations of SB on weekdays. Similar to what we have seen with PA, high SB is already apparent in preschoolers, and objective accelerometry data have shown children spending almost 8 hours per day in SB at age 4.4 years. ${ }^{15}$ Chen et al. ${ }^{1}$ reported a trend opposite from ours in the preschooler age group, with higher SB observed during non-school days rather than during school days. However, they did indicate that the lower SB on school days could be attributed to more regular naptimes scheduled by childcare centres, rather than an increase in $\mathrm{PA}^{1}$.

Our survey revealed that a significantly higher proportion of children $(18.8 \%)$ were receiving inadequate sleep on weekdays ( $<8$ hours of sleep) compared with on weekends, which concurs with previous observations in Singapore adolescents. ${ }^{37}$ Overall, evidence in preschoolers has shown that a large proportion $(>85.0 \%)$ were not meeting the sleep guidelines. ${ }^{12}$ The discrepancies in these findings between preschoolers and school-aged children could be due to the frequency and duration of naps the children were having in the day. The total sleep duration children in Singapore are receiving may be underestimated especially in younger children owing to the mandatory scheduled naptimes at childcare centres. It might be useful to account for the amount of sleep received at childcare, especially when approximately $70 \%$ of children in Singapore spend their days in full-day childcare by age 3 years, 
and this number increases to $89-91 \%$ in the subsequent years. ${ }^{38}$ While napping is more common in preschoolers, ${ }^{12} 37 \%$ of children in our study's age group were still taking daily naps that lasted for 1.5 hours on average on weekdays, and for 1 hour on weekends (data not shown), which is still a significant proportion compared with that in Western countries where only $0.9 \%$ of children still slept in the daytime by 7 years of age. ${ }^{39}$

Compared with previous studies, in which parents tend to underestimate the amount of sleep their children require, ${ }^{16,40}$ the parents in our study showed the most awareness for existing sleep guidelines (80\%). Only $30 \%$ were concerned about the amount of sleep their children were receiving, which aligns with approximately the $20 \%$ of children who were not meeting the recommended guidelines during the weekdays. Only half of the parents were aware of the PA guidelines for children, and only $11 \%$ were concerned about their child being overweight when the proxy-reported BMI showed that $18 \%$ of the children were overweight and $11 \%$ were obese. Our findings corroborate previous studies on how it is common for parents to underestimate the amount of PA their child requires ${ }^{41}$ and their child's weight status. ${ }^{42}$ In contrast, while parents in this study claimed to be most aware of the guidelines for recreational SVT (approximately 60\%) and were most concerned about SVT in children (approximately 70\%), the percentage of children exceeding the guidelines were still high. Along with the reported high use of screen devices among adult Singaporeans, ${ }^{35}$ a study by Bernard et al. has interestingly also shown that children's screen use behaviour at age 2-3 years was strongly influenced by parental behaviour. The results suggest that the presence of frequent screen users in the household is associated with children's screen behaviour, and thus targeting parental behaviour might be effective in reducing recreational SVT early in children. $^{14}$

\section{Strengths and limitations}

This study managed to collect comprehensive data on the lifestyle behaviours of children in Singapore aged 5-14 years old, along with data on parental perception on their child's health and parental awareness of existing guidelines. The strength of the study lies mainly in the use of a cost-effective and efficient online method of survey data collection, which was preferred over the conventional hard-copy survey. The limitations of the survey include selection bias and limitations in generalisability due to the survey method, which uses a convenience sampling method, and the small sample size. The wide age range sampled in this study may reduce the internal and external validity of the study. Other limitations include the possibility of underreporting, especially when parents had to report by proxy the children's lifestyle behaviours, such as SVT or PA, which might have taken place at school or when the parents are at work while the children are at home.

\section{CONCLUSION}

Findings from this study support the need for an integrated 24-hour activity guideline for children and adolescents in our Singapore population to help with better monitoring and improvement of these behaviours. The data from this study spurred the creation of the Singapore Integrated 24-Hour Activity Guidelines for Children And Adolescents. ${ }^{43}$ The next steps would be the dissemination and implementation of these guidelines in schools so that children and parents will be aware of integrated movement-related behaviours for the benefit of long-term health and for the prevention of obesity.

\begin{abstract}
Acknowledgements
We would like to thank members of the Singapore Integrated 24-Hour Activity Guidelines for Children and Adolescents Workgroup for their intellectual contribution to the development of the survey: Benny Kai Guo Loo, Benedict Chi'-Loong Tan, Michael Yong Hwa Chia, Poh Chong Chan, Dinesh Sirisena, Mohammad Ashik Zainuddin, Jean Yin Oh, Oon Hoe Teoh, Teresa Shu Zhen Tan, Micheal Chee Meng Lim, Ethel Jie Kai Lim, Falk Mueller-Riemenschneider, Ngiap Chuan Tan, Ratnaporn Siriamornsarp and Kee Chong Ng.

This research was supported by the Integrated Platform for Research in Advancing Metabolic Health Outcomes in Women and Children (IPRAMHO) - Singapore Ministry of Health's National Medical Research Council Centre Grant NMRC/CG/C008A/2017_KKH.
\end{abstract}

\section{REFERENCES}

1. Chen B, Bernard JY, Padmapriya N, et al. Associations between early-life screen viewing and 24 hour movement behaviours: findings from a longitudinal birth cohort study. Lancet Child Adolesc Health 2020;4:201-9.

2. Zhou Y, Aris IM, Tan SS, et al. Sleep duration and growth outcomes across the first two years of life in the GUSTO study. Sleep Med 2015;16:1281-6.

3. Padmapriya N, Aris IM, Tint MT, et al. Sex-specific longitudinal associations of screen viewing time in children at 2-3 years with adiposity at 3-5 years. Int J Obes (Lond) 2019;43:1334-43.

4. Janssen I, Leblanc AG. Systematic review of the health benefits of physical activity and fitness in school-aged children and youth. Int J Behav Nutr Phys Act 2010;7:40.

5. Chaput JP, Gray CE, Poitras VJ, et al. Systematic review of the relationships between sleep duration and health indicators in school-aged children and youth. Appl Physiol Nutr Metab 2016;41(6 Suppl 3):S266-82.

6. Atkin AJ, Gorely T, Clemes SA, et al. Methods of measurement in epidemiology: sedentary behaviour. Int J Epidemiol 2012; 41:1460-71.

7. Tremblay MS, Carson V, Chaput JP, et al. Canadian 24-Hour Movement Guidelines for Children and Youth: An Integration of 
Physical Activity, Sedentary Behaviour, and Sleep. Appl Physiol Nutr Metab 2016;41(6 Suppl 3):S311-27.

8. Yu Q, Aris IM, Tan KH, et al. Application and utility of continuous glucose monitoring in pregnancy: a systematic review. Front Endocrinol (Lausanne) 2019;10:697

9. Chia MYH, Tay LY, Chua TBK. Quality of life and meeting 24-h WHO guidelines among preschool children in Singapore. Early Childhood Educ J 2020;48:313-23.

10. Carson V, Janssen I. Volume, patterns, and types of sedentary behavior and cardio-metabolic health in children and adolescents: a cross-sectional study. BMC Public Health 2011;11:274.

11. Twenge JM, Campbell WK. Associations between screen time and lower psychological well-being among children and adolescents: evidence from a population-based study. Prev Med Rep 2018;12:271-83.

12. Chen B, Bernard JY, Padmapriya N, et al. Socio-demographic and maternal predictors of adherence to 24-hour movement guidelines in Singaporean children. Int J Behav Nutr Phys Act 2019;16:70.

13. Okely AD, Ghersi D, Hesketh KD, et al. A collaborative approach to adopting/adapting guidelines - The Australian 24-Hour Movement Guidelines for the early years (Birth to 5 years): an integration of physical activity, sedentary behavior, and sleep. BMC Public Health 2017;17(Suppl 5):869.

14. Bernard JY, Padmapriya N, Chen B, et al. Predictors of screen viewing time in young Singaporean children: the GUSTO cohort. Int J Behav Nutr Phys Act 2017;14:112.

15. Chen B, Waters $\mathrm{CN}$, Compier $\mathrm{T}$, et al. Understanding physical activity and sedentary behaviour among preschool-aged children in Singapore: a mixed-methods approach. BMJ Open 2020; 10:e30606

16. Aishworiya R, Chan P, Kiing J, et al. Sleep behaviour in a sample of preschool children in Singapore. Ann Acad Med Singap 2012; 41:99-104.

17. Goh SN, Teh LH, Tay WR, et al. Sociodemographic, home environment and parental influences on total and device-specific screen viewing in children aged 2 years and below: an observational study. BMJ Open 2016;6:e09113.

18. Chia M. Physical inactivity among children and adolescents in Singapore: a paradoxical issue. Acta Kinesiol 2008;2:7-15.

19. Lye JCT, Mukherjee S, Chia MYH. Physical activity and sedentary behavior patterns of Singaporean adolescents. J Phys Act Health 2015;12:1213-20

20. Yeo SC, Jos AM, Erwin C, et al. Associations of sleep duration on school nights with self-rated health, overweight, and depression symptoms in adolescents: problems and possible solutions. Sleep Med 2019;60:96-108.

21. Craig CL, Marshall AL, Sjostrom M, et al. International physical activity questionnaire: 12 -country reliability and validity. Med Sci Sports Exerc 2003;35:1381-95.

22. Owens JA, Maxim R, Nobile C, et al. Parental and self-report of sleep in children with attention-deficit/hyperactivity disorder. Arch Pediatr Adolesc Med 2000;154:549-55.

23. Owens JA, Spirito A, McGuinn M. The Children's Sleep Habits Questionnaire (CSHQ): psychometric properties of a survey instrument for school-aged children. Sleep 2000;23:1043-51.

24. Tremblay MS, Aubert S, Barnes JD, et al. Sedentary Behavior Research Network (SBRN) - Terminology Consensus Project process and outcome. Int J Behav Nutr Phys Act 2017;14:75.

25. Tremblay MS, LeBlanc AG, Kho ME, et al. Systematic review of sedentary behaviour and health indicators in school-aged children and youth. Int J Behav Nutr Phys Act 2011;8:98.
26. Crane JR, Naylor PJ, Temple VA. The physical activity and sedentary behaviour patterns of children in kindergarten and grade 2. Children (Basel) 2018;5:131

27. Paruthi S, Brooks LJ, D'Ambrosio C, et al. Recommended amount of sleep for pediatric populations: a consensus statement of the American Academy of Sleep Medicine. J Clin Sleep Med 2016;12:785-6.

28. World Health Organization. The Anthro Survey Analyser. Available at: https://worldhealthorg.shinyapps.io/anthro/. Accessed on 18 May 2021.

29. de Onis M, Onyango AW, Borghi E, et al. Development of a WHO growth reference for school-aged children and adolescents. Bull World Health Organ 2007;85:660-7.

30. Gilbey H, Gilbey M. The physical activity of Singapore primary school children as estimated by heart rate monitoring. Pediatr Exerc Sci 1995; 7:26-35.

31. Roman-Vinas B, Chaput JP, Katzmarzyk PT, et al. Proportion of children meeting recommendations for 24-hour movement guidelines and associations with adiposity in a 12-country study. Int J Behav Nutr Phys Act 2016;13:123.

32. The Straits Times. 12-year-olds in Singapore spend $61 \frac{1}{2}$ hours daily on electronic devices: survey, 2 April 2017. Available at: https://www.straitstimes.com/singapore/glued-to-screen-for-612hours-digital-habits-in-singapore. Accessed on 18 May 2021.

33. Engberg E, Figueiredo RAO, Rounge TB, et al. Heavy screen use on weekends in childhood predicts increased body mass index in adolescence: a three-year follow-up study. J Adolesc Health 2020;66:559-66

34. Sigmundova D, Badura P, Sigmund E, et al. Weekday-weekend variations in mother-/father-child physical activity and screen time relationship: a cross-sectional study in a random sample of Czech families with 5- to 12-year-old children. Eur J Sport Sci 2018;18:1158-67.

35. Google. Consumer barometer, November 2014. Available at: https://www.thinkwithgoogle.com/intl/en-145/consumer-insights/ consumer-journey/consumer-barometer. Accessed on 18 May 2021.

36. Hoffmann B, Kobel S, Wartha O, et al. High sedentary time in children is not only due to screen media use: a cross-sectional study. BMC Pediatr 2019;19:154

37. Lo JC, Lee SM, Lee XK, et al. Sustained benefits of delaying school start time on adolescent sleep and well-being. Sleep 2018;41:zsy052.

38. National Center on Education and the Economy. The early advantage: Singapore system at a glance, 14 October 2018. Available at: http://ncee.org/wp-content/uploads/2018/10/Singaporeat-a-Glance.pdf. Accessed on 18 May 2021.

39. Iglowstein I, Jenni OG, Molinari L, et al. Sleep duration from infancy to adolescence: reference values and generational trends. Pediatrics 2003;111:302-7.

40. Owens JA, Jones C, Nash R. Caregivers' knowledge, behavior, and attitudes regarding healthy sleep in young children. J Clin Sleep Med 2011;7:345-50.

41. Corder K, van Sluijs EM, McMinn AM, et al. Perception versus reality awareness of physical activity levels of British children. Am J Prev Med 2010;38:1-8.

42. Ruiter ELM, Saat JJEH, Molleman GRM, et al. Parents' underestimation of their child's weight status. Moderating factors and change over time: a cross-sectional study. PLoS One 2020;15: e0227761.

43. Loo BKG, Tan BCL, Chia MYH, et al. Singapore Integrated 24-Hour Activity Guidelines for Children and Adolescents. Singapore J Obstet Gynaecol 2021;52:20-31. 